\title{
Conocimiento Y Experiencia Del Estudiante De Enfermería En EI Manejo De Punzocortantes
}

\author{
Adriana Janeth Moreno Granados, Pasante en Servicio Social \\ Karla Jesús Ayala Bautista, Pasante en Servicio Social \\ Nayeli Rodríguez Dionisio, Pasante en Servicio Social
}

Licenciatura en Enfermería, Universidad Autónoma de Querétaro, México

Susana Angélica Dorantes Gómez, Licenciada en Enfermería Ruth Magdalena Gallegos Torres, Doctora en Ciencias de la Salud

Universidad Autónoma de Querétaro, México

Doi:10.19044/esj.2020.v16n27p76 URL:http://dx.doi.org/10.19044/esj.2020.v16n27p76

\section{Resumen:}

Introducción. Las prácticas profesionales durante el proceso de formación de los estudiantes de enfermería denotan una gran importancia, ya que es donde ellos construyen y reconstruyen los conocimientos adquiridos en el contexto áulico al reforzar así las medidas de seguridad en cada procedimiento realizado en el ámbito hospitalario y de esta manera evitar accidentes en especial con los objetos punzocortantes. Objetivo. Determinar el conocimiento y experiencia del estudiante de Enfermería sobre el manejo de punzocortantes durante la estancia clínica. Metodología. Estudio con enfoque cuantitativo transversal, descriptivo, realizado en la Universidad Autónoma de Querétaro con 202 estudiantes de sexo indistinto, a partir del tercer al octavo semestre, el instrumento constituido por 33 ítems validado por expertos y por pilotaje, para salvaguardar la identidad de los participantes firmaran el consentimiento informado. El proyecto fue registrado y evaluado ante un Comité de Investigación y Bioética. El análisis de los datos se realizó mediante estadística descriptiva. Resultados. La población su mayoría mujeres con el 86.1\% (174), en un rango de 18 a 34 años de edad. Más del $50 \%$ han recibido capacitación respecto al RPBI, y en base a la experiencia, el $35.1 \%$ (71) de los estudiantes han sufrido accidentes con objetos punzocortantes. Conclusión. La alta manipulación de punzocortantes entre los trabajadores de salud y estudiantes de enfermería trae consigo una gran cantidad de accidentes, los cuales son fuentes potenciales de enfermedades infectocontagiosas, es por ello que tanto el manejo y el deshecho de estos se debe realizar utilizando siempre las medidas de seguridad. 
Palabras Clave: Accidentes Y Eventos Con Materiales Peligrosos, Conocimientos, Estudiantes De Enfermería, Experiencia

\title{
Knowledge And Experience Of The Nursing Student In The Handling Of Sharps
}

\author{
Adriana Janeth Moreno Granados, Pasante en Servicio Social \\ Karla Jesús Ayala Bautista, Pasante en Servicio Social \\ Nayeli Rodríguez Dionisio, Pasante en Servicio Social
}

Licenciatura en Enfermería, Universidad Autónoma de Querétaro, México

Susana Angélica Dorantes Gómez, Licenciada en Enfermería Ruth Magdalena Gallegos Torres, Doctora en Ciencias de la Salud

Universidad Autónoma de Querétaro, México

\begin{abstract}
:
Introduction. The professional internships during the training process of nursing students are of great importance, since it is where they carry out the knowledge acquired at the educational institution thus reinforcing the safety measures in each procedure carried out in the hospital field and in this way avoid accidents especially with sharp objects. Objective. Determine the knowledge and experience of the Nursing student about the handling of sharps. Methodology. Transversal, descriptive study, carried out at the Autonomous University of Querétaro, in the Faculty of Nursing, located in the municipality of Queretaro. They were considered students of indistinct sex, from the third to the eighth semester, who agreed to participate in the study and signed informed consent. The project was registered and evaluated before a Research and Bioethics Committee. The analysis of the data was performed using descriptive statistics. Results. The population assessed consists of a total of 202 students, mostly women with $86.1 \%$ (174), a range of 18 to 34 years of age. Students were found to have medium knowledge and based on experience $35.1 \%$ (71) of students have suffered accidents with sharp objects. Conclusion. The high manipulation of sharps between health workers and nursing students brings with it a large number of accidents, which are potential sources of infectious diseases, is why both the management and waste of these must be carried out always using the safety measures.
\end{abstract}


Keywords: Accidents and Events with Hazardous Materials, Knowledge, Students, Nursing, Experience

\section{Introducción:}

El personal de la salud constituye uno de los grupos laborales con mayor número de riesgos ocupacionales. Los accidentes más frecuentes son las heridas por objetos punzocortantes, siendo generados por utensilios que pueden penetrar la piel o alguna otra parte del cuerpo, como: agujas, hojas de bisturí, ampolletas y catéteres, entre otros (Espinoza, K., Márquez, C. \& Sánchez., 2018).

Algunas de estas lesiones conllevan no solo a la incapacidad temporal, sino hasta el riesgo de transmisión de enfermedades $\mathrm{y}$ agentes infectocontagiosos, que pueden ser la hepatitis B, hepatitis C, Virus de Inmunodeficiencia Humana (VIH), tuberculosis, sífilis, malaria, herpes simple, difteria, Ébola, gonorrea cutánea, Staphylococcus aureus, Streptococcus pyogenes y Treponema pallidum (Gopar, Juárez, Cabello, Haro, \& Aguilar, 2015).

Se estima que alrededor del mundo una gran cantidad de trabajadores de la salud han sufrido accidentes con objetos punzocortantes. En América, el sector salud se constituye en cerca de 20 millones de trabajadores. En México, se calculan 1441000 personas que se desempeñan en el ámbito hospitalario mismas que pueden correr algún riesgo de sufrir un accidente por objetos punzocortantes, sin embargo, hasta ahora no se cuenta con un registro actualizado de incidencias (Gopar et al., 2015).

Al respecto, la Secretaría de Salud define al estudiante de enfermería como "aquel que se encuentra en proceso de formación en la carrera de enfermería de nivel medio superior o superior de alguna institución educativa perteneciente al Sistema Educativo Nacional" (Secretaria de Salud, 2013).

El estudiante de enfermería en proceso de formación, tiene como cualidad primordial, ser el depositario de un acervo cultural abundante, en el que se incluyen técnicas y procedimientos necesarios para llevarse a cabo en el medio hospitalario, sin embargo, a pesar de contar con conocimientos teóricos, es característica del estudiante también, carecer de las habilidades técnicas que permitan la implementación de estos en el área clínica (López, Hernández, Mendoza, Villarreal, \& Gasca, 2016).

En enfermería, la teoría y la práctica se complementan con la finalidad de acercar al estudiante con la realidad asistencial que le permita lograr un aprendizaje significativo, por esta razón, son insertados al ámbito sanitario por medio de prácticas profesionales en instituciones de salud pública, cursándolas a partir del segundo semestre de la Licenciatura en Enfermería (López, Hernández, Mendoza, Villarreal, \& Gasca, 2016). 
Los estudiantes de enfermería durante la realización de las prácticas, están expuestos a numerosos riesgos, pero uno de los más importantes por su frecuencia, gravedad y tendencia es el riesgo biológico, como pinchazos o cortes por objetos punzocortantes y salpicaduras oculares (Moreno-Arroyo et al., 2016).

El manejo de los objetos punzocortantes se debe realizar utilizando siempre medidas de barrera como los guantes. Los tubos capilares, navajas, lancetas, agujas de jeringas desechables, agujas hipodérmicas, agujas de sutura, bisturís y estiletes de catéter usados no se deben reencapsular, doblar o romper de forma manual. Tras su uso se deben eliminar en contenedores de RPBI (Residuos Peligrosos Biológico Infecciosos) punzocortantes (Duran, 2015).

Al respecto, los problemas del estudiante durante las prácticas profesionales, se destacan por el desconocimiento o falta de práctica en las medidas de bioseguridad, manejo y desecho de punzocortantes, teniendo como resultado, accidentes y heridas con objetos que lesionen la piel como ampolletas, agujas, lancetas, hojas de bisturí, entre otros (Charca, Huanca, \& Moreno, 2016).

Los accidentes con objetos punzocortantes son fuentes potenciales de enfermedades serias entre los trabajadores de salud y estudiantes de enfermería, donde estos últimos se vinculan con la institución durante varios semestres de práctica que posibilita la presencia de riesgos por la alta manipulación de estos elementos, así como el manejo de líquidos o fluidos corporales de pacientes sustancialmente comprometidos (Morelos, Ramírez, Sánchez, Chavarín, \& Meléndez, 2014).

Al respecto, en un estudio realizado sobre "Conocimiento del personal médico sobre el manejo de punzocortantes", aplicado en el Hospital General en Monterrey, N.L México, a enfermeras generales, auxiliares y jefas de piso, se destacó que el personal encuestado tuvo el siguiente nivel de conocimiento: bueno el $17 \%$, regular el $35 \%$, deficiente el $48 \%$. Se identificó que la mitad del personal no lleva un manejo adecuado de punzocortantes y el resto se encuentra de regular a bueno (Carreño, Hernández, Loaiza, \& Arguelles, 2015).

En una investigación acerca de la "Relación entre nivel de conocimiento y manejo de los residuos bio-contaminados, y contaminación generada en dos clínicas odontológicas universitarias", en la Universidad Nacional Mayor de San Marcos y la Universidad Nacional Federico Villarreal, se observó que ambas instituciones tienen un adecuado manejo en objetos punzocortantes (Saucedo \& Soto, 2013).

Se encuentra otro estudio acerca del "Conocimiento y cumplimiento de las medidas de bioseguridad y accidentes biológicos de los estudiantes de enfermería en las prácticas clínicas". Dentro de los resultados de este estudio 
durante la práctica clínica, se encontró que los estudiantes realizan las medidas de bioseguridad en los siguientes porcentajes: el 60,2\% aplica las normas de higiene personal, un $66,1 \%$ hacen uso de protección de barrera y $44 \%$ manifiesta el manejo en la disposición de objetos punzocortantes (Merino, Dura, Rodríguez, \& Gonzales, 2014).

De otro estudio denominado "Nivel de conocimiento y aplicación de las medidas de bioseguridad del personal de enfermería", se manifestó que, en el ámbito de manejo de objetos punzocortantes, el personal de enfermería realiza reencauchado de agujas, por lo tanto, se evidencia que la disposición de este material es deficiente con un $64 \%$, regular con un $27 \%$ y bueno representado en un 9\% (Bautista et al., 2013).

Con base en lo anteriormente planteado es que se llevó a cabo la presente investigación con la finalidad de determinar el conocimiento y experiencia del estudiante de enfermería sobre el manejo de punzocortantes en la Universidad Autónoma de Querétaro, a través de la aplicación de un cuestionario, de manera tal que se pueda contar con un referente actual del fenómeno que permita contribuir a la educación de los estudiantes y a la prevención de enfermedades infectocontagiosas por accidentes con objetos punzocortantes.

\section{Metodología:}

Se trató de un estudio con enfoque cuantitativo, de tipo descriptivo, transversal (Hernández, 2014), que se realizó en la Facultad de Enfermería de la Universidad Autónoma de Querétaro, la población fue de 421 estudiantes del campus Centro Universitario, los cuales se dividen en diferentes semestres que van desde primero hasta octavo de forma presencial y realizan prácticas hospitalarias a partir de segundo semestre, por lo cual para este estudio se consideraron a los estudiantes de tercer a octavo semestre.

El muestreo fue probabilístico; se aplicó la fórmula para poblaciones finitas, aplicando el muestreo aleatorio estratificado proporcional (Casal \& Mateu, 2003), obteniendo un total de la muestra de 202 estudiantes para aplicar la herramienta de recolección de datos.

El estudio se aprobó ante el Comité de Investigación y un Comité de Bioética de la Facultad de Enfermería, mismos que evaluaron los términos de los principios éticos, de acuerdo a los lineamientos de la Ley General de Salud en materia de Investigación. Los estudiantes fueron seleccionados de acuerdo al cumplimiento de los siguientes criterios de selección:

- Inclusión: Todos los estudiantes que mediante el consentimiento informado aceptaran participar en el presente estudio.

- Exclusión: estudiantes de otro campus, ya que la facultad tiene 3. 
- Eliminación: Los cuestionarios de los estudiantes que no estuvieran presentes al momento del levantamiento de la información y aquellos que no fueron contestados al $100 \%$.

Se elaboró una herramienta de recolección de datos titulada "Cuestionario sobre conocimientos y experiencia en el manejo de objetos punzocortantes", el cual consta de 33 ítems distribuidos en 3 secciones. En la primera se encuentran los datos sociodemográficos, la segunda mide el nivel de conocimientos de acuerdo a la NOM-087-ECOL-SSA1-2002 (Secretaría de Salud, 2002) y en la tercera permite identificar si los participantes han estado expuestos a accidentes con objetos punzocortantes alguna vez.

Dicho cuestionario fue sometido a método Delphi para su validación, en la cual participaron 3 expertos a quienes se les invitó, por medio de una carta personalizada, en donde se mencionaba cuál era el aparatado o los apartados a evaluar del formato, quedando integrados los evaluadores de la siguiente manera:

- Una Maestra en Ciencias, evaluando la metodología y estructura para el análisis de las preguntas,

- Una Enfermera Especialista, evaluando las preguntas desde el ámbito de la Salud Pública, y

- Un Licenciado en Medicina, evaluando que el apartado de RPBI se encontrara apegado a la Norma Oficial Mexicana NOM-087.

El proceso de validación se realizó en 2 fases que se describen a continuación:

- En la primera fase se envió un documento a los 3 expertos que incluía el título de protocolo de investigación, los objetivos del estudio (general y específicos), justificación y el cuestionario a evaluar el cual contaba con 22 preguntas. En ella dos de los validadores aprobaron el cuestionario, mientras una de ellas mencionó que se tenían que agregar preguntas sobre la experiencia y el conocimiento, aunado a esto, se agregaron incisos a las preguntas ya que sólo se encontraban espacios para marcar la respuesta correcta.

- En la segunda fase, debido a la incorporación de 11 preguntas, se volvió a enviar a los expertos el cuestionario para una nueva revisión, donde aprobaron el cuestionario ya que se habían realizado los cambios sugeridos, quedando así un cuestionario final con 33 preguntas.

Al finalizar el proceso de validación, en el mes de Noviembre del año 2019 se llevó acabo la prueba piloto con los estudiantes de octavo semestre pertenecientes al periodo escolar 19-2, los cuales egresaron el mismo año, y en el mes de Marzo del año 2020 se realizó la prueba definitiva, la cual incluía a los alumnos de tercero a octavo semestre del periodo escolar 20-1. 
Los resultados de la prueba piloto arrojaron que los estudiantes no tenían complicaciones para comprender el cuestionario ni se encontró la necesidad de realizar ajustes al mismo.

Los datos se analizaron utilizando estadística descriptiva mediante el manejo de frecuencias y desviación estándar.

\section{Resultados:}

La población en estudio estuvo integrada por un total de 202 estudiantes, siendo la mayoría mujeres con un $86.1 \%$ y el $13.9 \%$ hombres, con una edad promedio de 23 años, la edad mínima fue de 18 años y máxima de 34 años, con una desviación estándar (DS) de 1.802. Con respecto al estado civil, el $94.1 \%$ de la muestra es soltero, $2.5 \%$ casado, $2 \%$ en unión libre. En cuanto al semestre en que se encuentran, los datos se muestran en la tabla 1.

Tabla 1. Semestre en el que se encuentran ubicados los estudiantes de enfermería, Universidad Autónoma de Querétaro, 2020.

\begin{tabular}{ccc}
\hline Valores & $\begin{array}{c}\text { Frecuencia } \\
\text { (fi) }\end{array}$ & $\begin{array}{c}\text { Porcentaje } \\
\text { (f \%) }\end{array}$ \\
\hline Tercer semestre & 30 & 14.9 \\
cuarto semestre & 40 & 19.8 \\
Quinto semestre & 22 & 10.9 \\
Sexto semestre & 41 & 20.3 \\
Séptimo semestre & 26 & 12.9 \\
Octavo semestre & 43 & 21.3 \\
Total & 202 & $100 \%$ \\
\hline
\end{tabular}

Fuente: cuestionario

El $61.4 \%$ pertenece al turno matutino y $38.6 \%$ al vespertino.

Con respecto a si los estudiantes han recibido capacitación sobre los RPBI, el $56.9 \%$ dijeron que sí la recibieron en el aula de la institución educativa, así como en la unidad hospitalaria a lo largo de sus prácticas.

El 59.4\% de los participantes identifican que los materiales generados durante los servicios de atención médica que contengan agentes biológicoinfecciosos y que pueden causar efectos nocivos a la salud y al ambiente, son los RPBI. El 54\% señaló que los residuos no natómicos no se consideran como materiales peligrosos.

La totalidad de la población en estudio sabe lo que es un objeto punzocortante. El $81.2 \%$ de los estudiantes mencionaron que cultivos y cepas son aquellos que han estado en contacto con fluidos humanos o animales o sus muestras biológicas durante el diagnóstico y tratamiento.

Referente al conocimiento de las fases del manejo de RPBI, la información se muestra en la tabla 2.

Tabla 2. Selección de la fase que no pertenece al manejo de RPBI. Estudiantes de enfermería, Universidad Autónoma de Querétaro, 2020. 


\begin{tabular}{ccc}
\hline & (fi) & \\
\hline Identificación de los residuos & 28 & 13.93 \\
Envasado de los residuos & 34 & 16.8 \\
$\begin{array}{c}\text { generados } \\
\text { Traslado de los residuos por } \\
\text { vías RPBI (Respuesta } \\
\text { correcta) }\end{array}$ & 10 & 5.0 \\
Almacenamiento temporal & & \\
Recolección y trasporte & 31 & 15.3 \\
$\quad$ externo & 8 & 4.0 \\
Tratamiento & 79 & 39.1 \\
Disposición final & 12 & 5.9 \\
Total & 202 & 100.0 \\
\hline
\end{tabular}

Fuente: cuestionario

En la tabla 2 se observa que el $39.1 \%$ de los encuestados menciona que el "tratamiento" no es una fase del manejo de los RPBI, donde efectivamente sí es una etapa es el proceso, en el cual se trata los RPBI por métodos físicos o químicos para la eliminación de microorganismos patógenos; el $5.0 \%$ de la muestra está en lo correcto al indicar que el "traslado de los residuos por vías RPBI" no es una fase del manejo.

Con respecto a cuáles se consideran objetos punzocortantes, el $63.9 \%$ manifiesta que los tubos capilares no son punzocortantes, sin embargo, de acuerdo a la norma sí lo es, debido que ha tenido contacto con pacientes en el diagnóstico y tratamiento de este.

En relación a los recipientes de envasado de los RPBI punzocortantes, que la población en estudio refiere como apropiados, los datos se muestran en la tabla 3.

Tabla 3. Frecuencia de datos correctos acerca del envasado de los RPBI punzocortantes. Estudiantes de enfermería, Universidad Autónoma de Querétaro, 2020.

\begin{tabular}{ccc} 
Valores & $\begin{array}{c}\text { Frecuencia, de elección } \\
\text { de respuesta } \\
\text { (fi) }\end{array}$ & $\begin{array}{c}\text { Porcentaje } \\
\text { (f \%) }\end{array}$ \\
\hline $\begin{array}{c}\text { Recipiente hermético de color } \\
\text { rojo. (Incorrecto) }\end{array}$ & 80 & 39.6 \\
$\begin{array}{c}\text { Recipiente hermético de color } \\
\text { amarillo (Incorrecto) }\end{array}$ & 2 & 1.0 \\
$\begin{array}{c}\text { Recipiente rígido de propileno } \\
\text { rojo. (Correcto) } \\
\text { Total }\end{array}$ & 120 & 59.4 \\
\hline
\end{tabular}

Fuente: cuestionario

En la tabla 3 se hace notar que el 59.4\% de los estudiantes señalan que los recipientes rígidos de propileno color rojo son los correctos para el desecho 
de objetos punzocortantes, lo cual es acertado, y a su vez está establecido en la NOM-087-ECOL-SSA1-2002 sobre el manejo de RPBI.

La mayoría de los encuestados (89.6\%) tienen el conocimiento de la capacidad límite del llenado de los recipientes para los RPBI punzocortantes, mencionando que se puede llenar hasta su $80 \%$.

En relación al conocimiento del tiempo de almacenamiento temporal para los recipientes de los RPBI punzocortante, el 33.7\% de la población en estudio tiene desconocimiento, mientras que el $26.2 \%$ mencionó que este periodo es de máximo 30 días, el 24.3\% máximo 15 días y el 15.8\% máximo 7 días. El tiempo correcto para el almacenamiento temporal es de máximo 15 días.

La mayoría de los estudiantes (56.4\%) tienen conocimiento acerca del tratamiento y disposición final de los RPBI punzocortantes mencionando que se trata de ser inhumados o incinerados y desechados en sitios autorizados.

Con respecto al lugar donde han cursado prácticas profesionales, los datos se muestran en la tabla 4.

Tabla 4. Instituciones donde se han cursado prácticas profesionales los estudiantes de enfermería de la Universidad Autónoma de Querétaro, 2020

\begin{tabular}{|c|c|c|}
\hline Valores & $\begin{array}{l}\text { Frecuencia } \\
\text { (fi) }\end{array}$ & $\begin{array}{c}\text { Porcentaje } \\
\text { (f \%) }\end{array}$ \\
\hline Instituto Mexicano del & 84 & 41.6 \\
\hline Seguro Social (IMSS), & & \\
\hline $\begin{array}{l}\text { Servicios de Salud del } \\
\text { Estado de Querétaro } \\
\text { (SESEQ) }\end{array}$ & 74 & 36.6 \\
\hline $\begin{array}{c}\text { Instituto de Seguridad y } \\
\text { Servicios Sociales de los } \\
\text { Trabajadores del Estado } \\
\text { (ISSSTE) }\end{array}$ & 26 & 12.9 .9 \\
\hline $\begin{array}{l}\text { Hospital de Especialidades } \\
\text { del Niño y la Mujer } \\
\text { (HENM) }\end{array}$ & 18 & 8.9 \\
\hline Total & 202 & $100 \%$ \\
\hline
\end{tabular}

Fuente: cuestionario

Los objetos punzocortantes con mayor contacto entre los estudiantes durante sus labores intrahospitalarias se muestran en la tabla 5.

Tabla 5. Objetos punzocortantes con los que están en contacto los estudiantes de enfermería durante las prácticas profesionales, Universidad Autónoma de Querétaro, 2020.

\begin{tabular}{ccc}
\hline Valores & $\begin{array}{c}\text { Frecuencia } \\
\text { (fi) }\end{array}$ & $\begin{array}{c}\text { Porcentaje } \\
\text { (f \%) }\end{array}$ \\
\hline Tubos capilares & 7 & 3.5 \\
Navajas & 24 & 11.9 \\
Lancetas & 40 & 19.8
\end{tabular}


Agujas de jeringas desechables

Agujas hipodérmicas

Suturas

Bisturí

Estilete de catéter
23.8

16.3

11.4

6.4

6.9

Total

202

100.0

Fuente: cuestionario

En la tabla 5 se observa que el $23.8 \%$ de la población en estudio señala que tiene mayor contacto con agujas de jeringas desechables, seguido de lancetas con $19.8 \%$, y el $16.3 \%$ con agujas hipodérmicas.

La totalidad de los encuestados mencionaron haber realizado preparación y ministración de medicamentos, así como la realización de procedimientos invasivos durante sus prácticas profesionales, donde el $35.1 \%$ sufrieron algún accidente con objeto punzocortante de los cuales, el 97.2\% tuvieron de 1 a 3 accidentes desde el inicio de su formación. El 42.3\% de la población en estudio refiere que dicho accidente fue con lancetas, seguido de $29.6 \%$ con agujas hipodérmicas. El 59.2\% de los accidentes ocurrieron antes de realizar algún procedimiento, el $38.0 \%$ después del receso de la jornada de prácticas y el $46.5 \%$ refiere haber ocurrido en el periodo intermedio de sus prácticas profesionales.

De los estudiantes que mencionaron si han sufrido algún accidente con algún objeto punzocortante, el $80.3 \%$ refiere haber realizado como acción post-exposición el permitir el sangrado exprimiendo la herida y lavado con abundante agua y jabón, de los cuales solo el $67.1 \%$ de los accidentes fueron reportados, con un $57.4 \%$ al personal en turno.

\section{Conclusión y discusión:}

Como parte de los datos obtenidos del estudio, se encontró que el $56.9 \%$ de los participantes han recibido información y/o capacitación sobre el fenómeno, tanto en la Facultad de Enfermería como en una institución hospitalaria y tan solo 3 de cada 10 alumnos tuvo algún accidente con objetos punzocortantes. Los datos anteriores difieren con lo reportado por Carreño, Hernández, Loaiza y Arguelles en su investigación de 2015, donde se destaca que el personal tiene un nivel de conocimiento deficiente con el $48 \%$, lo que favorece que no se lleve un manejo adecuado de punzocortantes.

Los estudiantes de enfermería realizan procedimientos para la preparación y ministración de medicamentos, así, como la ejecución de procedimientos invasivos durante sus prácticas profesionales. Al respecto, el $59.4 \%$ de los participantes identifica claramente que los materiales generados durante los servicios de atención médica que contengan agentes biológico- 
infecciosos y que pueden causar efectos nocivos a la salud y al ambiente, son RPBI. 5.4 de cada 10 alumnos señalan que los residuos no anatómicos no se consideran como RPBI. Sin embargo lo plasmado en la Norma Oficial Mexicana NOM-087-ECOL-SSA1-2002 (Secretaría de Salud, 2002) para los Residuos peligrosos biológico-infecciosos, considera que los recipientes desechables que contengan sangre líquida, los materiales de curación, empapados, saturados, o goteando sangre o cualquiera de los siguientes fluidos corporales: líquido sinovial, líquido pericárdico, líquido pleural, líquido céfalo-raquídeo o líquido peritoneal, de pacientes con sospecha o diagnóstico de enfermedad infecciosa son residuos con alto material infectocontagioso.

Los alumnos encuestados mencionaron sí saber lo que es un objeto punzocortante, pero sólo el $18.8 \%$ refiere que los objetos punzocortantes son "aquellos que han estado en contacto con humanos o animales o sus muestras biológicas durante el diagnóstico y tratamiento". E1 81.2\% restante, menciona que lo anterior corresponde a cultivos y cepas. La Norma Oficial Mexicana NOM-087-ECOL-SSA1-2002 (Secretaría de Salud, 2002), define a los cultivos y cepas de agentes biológico-infecciosos como "los utensilios desechables usados para contener, transferir, inocular y mezclar cultivos de agentes biológico-infecciosos".

Con respecto al manejo de RPBI punzocortantes, el $0.5 \%$ correspondiente a solo 10 de los participantes, declararon que el "traslado de los residuos por vías RPBI" no corresponde a una de las fases para el manejo de RPBI, a lo que la Norma Oficial Mexicana para Los Residuos Peligrosos Biológico-Infecciosos, reconoce a la Identificación de los residuos, al Envasado de los residuos generados, al Almacenamiento temporal, a la Recolección y transporte externo, al Tratamiento y Disposición final como las fases del Manejo de residuos peligrosos biológico-infecciosos.

En relación a la identificación de los objetos punzocortantes el 63.9\% manifiesta que los tubos capilares no son considerados como punzocortantes. En contraste a lo anterior en la Norma Oficial Mexicana NOM-087 (Secretaría de Salud, 2002), clasifica a los tubos capilares, navajas, lancetas, agujas de jeringas desechables, agujas hipodérmicas, de sutura, de acupuntura y para tatuaje, bisturís y estiletes de catéter, como objetos punzocortantes.

Con respecto a las experiencias, el 35.1\% sufrieron algún accidente con un objeto punzocortante, siendo las lancetas las de mayor predominancia, seguido de las agujas hipodérmicas y después las jeringas desechables. 6 de cada 10 accidentes ocurridos fueron antes de realizar algún procedimiento, por lo que acontece de bajo riesgo, y en su mayoría estos fueron reportados al personal en turno. Lo referido es apoyado por Charca, Huanca y Moreno en su estudio realizado en 2016, donde mencionan que los problemas de los estudiantes durante sus prácticas profesionales, se destacan por el desconocimiento o falta de práctica en las medidas de bioseguridad, manejo y 
desecho de punzocortantes, teniendo como resultado, accidentes y heridas con objetos que lesionen la piel como ampolletas, agujas, lancetas, hojas de bisturí, entre otros.

Con base al estudio realizado, se concluye que el conocimiento y experiencia del estudiante de enfermería en el manejo de punzocortantes es medio. Lo anterior sugiere la necesidad de reforzar el conocimiento, ya que una parte de los encuestados se encuentra desacertado en la clasificación de los Residuos Peligrosos Biológico-Infecciosos e ignora los procedimientos fundamentales del manejo (fases) y disposición adecuada, referente al desecho de los diferentes punzocortantes, en la capacidad y uso del contenedor.

Es imprescindible fortalecer los rubros débiles detectados a través de un programa de capacitación continua en el manejo adecuado de objetos punzocortantes con el fin de disminuir riesgos de accidentes biológicos e incrementar su conocimiento, con las indicaciones establecidas en la norma de bioseguridad. A su vez también realizar evaluaciones periódicas para evaluar el conocimiento de los estudiantes y eficacia del programa.

Es necesario aportar a los estudiantes, asignaturas teóricas y prácticas dirigidas a la manipulación de estos materiales, donde mejoraran su desempeño y reforzaran sus habilidades favoreciendo al ejercicio de sus prácticas profesionales.

Se promueve la implementación de las precauciones estándar y universales; la eliminación del reencapuchado, la manipulación con pinzas de objetos punzocortantes y la disposición en recipientes rígidos, inmediatamente después de su uso, el suministro y uso de equipos de protección personal, la inmunización con el cumplimiento del esquema de vacunación y la profilaxis pos-exposició, con el fin de prevenir cualquier tipo de enfermedad infecciosa tras una punción.

\section{References:}

1. Bautista, L. M., Delgado, C. C., Hernández, Z. F., Sanguino, F. E., Cuevas, M. L., Arias, Y. T., \& Mojica, I. C. (2013). Nivel de conocimiento y aplicación de las medidas de bioseguridad del personal de enfermería. Revista Ciencia y Cuidado, ISSN 1794- 9831, Vol. 10, No. 2, 2013, Págs. 127-135, 10(2), 127-135.

2. Carreño, J., Hernández, H., Loaiza, N., \& Arguelles, L. (2015). Conocimiento del personal médico sobre el manejo de punzocortantes. Revista de Divulgación Científica y Tecnológica de La Universidad Autónoma de Nuevo León, 71, 135. https://doi.org/2007-1175

3. Casal, J., \& Mateu, E. (2003). Tipos de muestreo. Revista Epidemiológica Y Medicina Preventiva, 1 (1), 3-7. Retrieved from http://www.mat.uson.mx/ ftapia/Lecturas\%20Adicionales\%20(Cóm o\%20diseñar\%20una\%20encuesta)/TiposMuestreo1.pdf 
4. Charca, L. C., Huanca, G. H., \& Moreno, O. (2016). Accidentes biológicos en estudiantes del último año de medicina de tres hospitales de Lima, Perú. Medwave, 16(07), e6514-e6514. https://doi.org/10.5867/medwave.2016.07.6514

5. Duran, O. (2015). Acciones que debe tomar el personal de salud para la prevención de accidentes laborales por pinchazos. Tesis.

6. Espinoza, K. Márquez, C. Sánchez, S. (2018). Factores que predisponen a la exposición de accidentes punzocortantes en enfermeras, del servicio de centro Quirúrgico de un Hospital de Lima Metropolitana. III, 224-234.

7. Gopar, R., Juárez, C., Cabello, A., Haro, L., \& Aguilar, G. (2015). Panorama de heridas por objetos punzocortantes en trabajadores intrahospitalarios. Rev Med Inst Mex Seguro Soc, 53(3), 356-361. Retrieved from http://www.medigraphic.com/pdfs/imss/im2015/im153q.pdf

8. Hernández Sampieri, R., Fernández Collado, C., Baptista Lucio, P. (2014).

Metodología de la Investigación. (6 ${ }^{\text {a }}$ Ed). México, D.F. McGraw-Hill., Pág. 155. Metodología de la Investigación. (6 ${ }^{a}$. Ed). México, D.F. McGraw-Hill., Pág. 155.

9. López, V., Hernández, M. A., Mendoza, A., Villarreal, E., \& Gasca, M. A. (2016). Apoyo a estudiantes de enfermería en su primera práctica clínica ante situaciones de estrés. Revista de Enfermería Del Instituto Mexicano Del Seguro Social, 24(3), 165-170.

10. Merino, F., Dura, M., Rodríguez, E., \& Gonzales, S. (2014). Conocimiento y cumplimiento de las medidas de bioseguridad y accidentes biológicos de los estudiantes de enfermería en las prácticas clínicas. Servier, 10.

11. Morelos, R., Ramírez, M., Sánchez, G., Chavarín, C., \& Meléndez, E. (2014). El trabajador de la salud y el riesgo de enfermedades infecciosas adquiridas. Revista de La Facultad de Medicina de La UNAM, 34-42.

12. Moreno-Arroyo, M. C., Puig-Llobet, M., Falco-Pegueroles, A., LluchCanut, M. T., García, I. C., \& Roldán-Merino, J. (2016). ¿Qué percepción del riesgo biológico tienen los estudiantes de Grado de Enfermería? Revista Latino-Americana de Enfermagem, 24. https://doi.org/10.1590/1518-8345.0722.2715

13. Orozco, A., Nuñez, I., Figueroa, O., \& Ochoa, A. (2015). Factores asociados a la profesionalización de Enfermería en México. Revista CONAMED, 20(S1), S41-S47. Retrieved from https://www.medigraphic.com/cgibin/new/resumen.cgi?IDARTICULO=7958 
14. Saucedo, A., \& Soto, V. (2013). Conocimientos y prácticas de bioseguridad en internos de medicina humana en hospitales de Lambayeque. 6(4), 17-21.

15. Secretaria de salud. (2013). ESTADO DE LA ENFERMERÍA EN MÉXICO (Primera ed). Ciudad de México

16. Secretaria de Salud. (2002). NORMA Oficial Mexicana NOM-087ECOL-SSA1-2002, Protección ambiental - Salud ambiental - Residuos peligrosos biológico-infecciosos- Clasificación y especificaciones de manejo. Diario Oficial de La Federación. Retrieved from http://www.salud.gob.mx/unidades/cdi/nom/087ecolssa.html 